\title{
Entropy production in hydrodynamic sliding stream magnetic nano- fluid on a porous expanded sheet
}

\author{
Ali Salimi ${ }^{1}$, Arash Shams Taleghani ${ }^{2 *}$ \\ 1- Department of Mechanical Engineering, Brojerd Branch, Islamic Azad university, Brojerd, Iran \\ 2- Aerospace Research Institute, Ministry of Science, Research and Technology, Tehran, Iran
}

\begin{abstract}
This study aims to study the fluid flow, heat transfer and entropy production on a porous expanded rapidly in Nano-fluids in the attendance slip boundary condition numerically. In this regard, various metals will be considered as nanoparticles with water as base fluid. Nanofluid that the distribution of nanoscale particles in conventional fluid caused by a new generation of fluids with great potential in industrial applications. The effects of various physical parameters of the problem was examined, such as the volume fraction of nanoparticles, different types of nanoparticles, magnetic parameters, parameter sliding speed, and suction parameters on curves speed and temperature and entropy production. The results show that there was an increase in the entropy production with increasing volume fraction of nanoparticles in heat transfer and thermal boundary layer thickness.
\end{abstract}

Keywords: the second law of thermodynamics, sliding flow, nano-fluids, magnetic hydrodynamic flow, expanded sheet.

\section{Introduction}

Prodigious consumption of energy resources in the last half century has led to the world's energy policies review. The governments have adopted significant measures to eliminate wasted energy, promoting energy efficiency policies and technologies in buildings, appliances, transportation and industry. Academies and researchers have started a closer look at the energy conversion systems, the development of new techniques and methods of analysis in order to better use of resources and environmental protection.

The purpose of the above concepts is to create due to increased efficiency in all types of production systems, conversion and energy consumption. Measuring the efficiency of heat exchange systems is very limited to the first law of thermodynamics. However, on the basis of calculations using second law of thermodynamics, entropy production is highly reliable than the calculations based on the first law of thermodynamics. Thermodynamic entropy production is related to irreversible processes in all common heat transfer. Various factors cause the entropy production such as heat transfer in the narrow temperature gradient, convective heat transfer characteristics, and the effect of viscous and so on. Since the production of entropy is a measure of the measurement system available job destruction, its

\footnotetext{
*Electronic address : Taleghani@ari.ac.ir
} 
decline is the result of optimum design of energy systems [1]. Also, the entropy production cycle leads to reduce power output or increase the input power refrigeration cycle. Therefore, accurate calculations of entropy production plays an important role in the development of components such as heat exchangers, pumps, turbines, piping networks, energy storage systems and electronic cooling equipment.

Bijan [2] focused on various factors in the production of entropy in thermal engineering applications. Bijan [3] expressed the second law of thermodynamics statements using the forced displacement of issues, as well as established the idea of entropy production number and distribution of irreversibly. After that, a lot of research was performed to determine the entropy production and irreversibility for combinations of geometric curves, flow conditions and different boundary conditions. According to the non-linear nature of entropy production issues, little analytical work has been done in this area. For example, Mahmoud and Fraser [4] studied the second law in several fundamental issue heat transfers and in any case, have made the analytical expressions for the entropy production number. These issues on a steady laminar flow incompressible fluid in the channel with a circular cross section, so that the channel is made up of two parallel systems. Entropy production induced by heat transfer analysis and flow between rotating cylinders, few studies have been done that pointed out a few of them.

Yilbaz [5] presented the analysis of isothermal entropy boundary condition for a rotating outer cylinder under different warming in a loop. The effects of entropy production were ignored due to fluid friction in its analysis. Mahmoud and Fraser [6] studied the entropy production in a cylindrical gap with constant flux boundary condition. They analyzed the conservation equations in cylindrical coordinates to obtain the analytical expression for entropy production and Bijan simplified dimensionless numbers as a function of the parameters involved in the issue. Their results indicate that this is the minimum amount the average rate of production of entropy, when there is no relative motion between the two cylinders and both cylinders rotate in one direction at the same speed occurs. In another work [7] the entropy production is fixed between the cylinder with a relative rotation where the cylinder internal temperature and external temperature or pressure cylinder were studied. It has been shown that the minimum average rate of entropy production depends only on the ratio of the radius, and when the value of angular velocity equals to the value of the radius, is the minimum amount. Mirzazadeh and colleagues [8] examined the entropy production induced by heat transfer and fluid flow is a non-linear viscoelastic between rotating concentric cylinders. Their results showed that the total entropy production number decreases with increasing the elasticity of the fluid, and increases as the Brinkman number increases.

The development of nanotechnology and nano-sized particles makes it possible to mix the particles of the size of the fluid. Based on the description and less problems nanoparticles, the particles are superior to the particles with a micrometer and millimeter dimensions. In fact, the small size of the particles and the low volume fraction is used, and solves problems such as corrosion and pressure drop. One of the most important factors in choosing fluids is the stability of nano-particles in the fluid in the heat exchange. Finally, the smaller the particles are, however, also increases the relative level of heat transfer. As a result, the thermal 
efficiency of particulate matter is a function of the heat transfer surfaces that increases by reducing the particle size.

Choi and Eastman were the first people in Argune National Laboratory in the United States, called the suspension of nanoparticles in nano-fluids base fluid and showed an extraordinary increase in heat transfer coefficient [9]. Experimental results show that it is adding 1 to 5\% by volume particle mass, thermal conductivity of the mixture can increase up to $20 \%$ compared with the base fluid $[10,11]$. This increase in heat transfer cannot be attributed exclusively to the thermal conductivity of the nanoparticles. Other mechanisms also have a direct impact such as particle density, volume fraction, Brownian motion, particle size, shape and particle surface area increases heat transfer. Mustafa et al [12] using homotopy analysis and taking into account the effect of Brownian motion, examined the nano-fluid flow stagnation point on an extension. Khalili and colleagues [13] examined MHD unsteady flow and heat transfer near the point of inertia on expanding the presence of nanofluids. Ziaee rod and Kasaeipoor [14] studied solving the boundary layer flows parallel hybrid copper water nanofluids passed over a horizontal flat-screen numerically using finite difference method Klrbaks.

Since the second law of thermodynamics analysis is reliable, it is an analysis of the first law of thermodynamics, the second law of thermodynamics of fluid flow and heat transfer is studied along on a permeable expanded rapidly in nanofluids in the attendance slip boundary condition Runge-Kutta numerical method -the study of heat transfer in the flow drawn much attention due to the wide applications in chemical engineering respectively.

\section{Description of the problem and governing equations}

Laminar flow incompressible fluid of dynamics of Nano-fluids is considered in the presence of external magnetic field uniform vertical permeable expanded vertically on a sheet. The sheet is powered by two different -oriented and equal force and spreads in order to apply, (the center is kept constant) along with $x$. Speed of the sheet is assumed as $u_{w}(x)=a x$. The viscous dissipation is ignored. Physical geometry and coordinate system are given in Fig. 1. The conservation equations of continuity, momentum and energy for incompressible flow boundary layers in a quiet MHD nanofluids is as follows:

$$
\begin{aligned}
& \frac{\partial u}{\partial x}+\frac{\partial v}{\partial y}=0, \\
& \rho_{n f}\left(u \frac{\partial u}{\partial x}+v \frac{\partial u}{\partial y}\right)=\mu_{n f} \frac{\partial^{2} u}{\partial y^{2}}-\sigma B^{2} u, \\
& \left(\rho c_{p}\right)_{n f}\left(u \frac{\partial T}{\partial x}+v \frac{\partial T}{\partial y}\right)=k_{n f} \frac{\partial^{2} T}{\partial y^{2}},
\end{aligned}
$$

In above equations, $u$ and $v$ are the high-speed components along with electrical distribution coefficient $x, y$ and $\sigma, B$ is uniform magnetic field, $c_{p}$ is the specific heat at constant 
pressure, $k$ is thermal conductivity and $T$ is the temperature of fluid. In the above equations, $\mu_{n f}$ and $\rho_{n f}$ are dynamic viscosity and density of nanofluids, which $\mu_{n f}$ is presented by Brinkman [15] as follows, and as well as $k_{n f}$ is the effective thermal conductivity of nanofluids and $\left(\rho c_{p}\right)_{n f}$ is thermal capacity of nanofluids:

$\left(\rho c_{p}\right)_{n f}=(1-\varphi)\left(\rho c_{p}\right)_{f}+\varphi\left(\rho c_{p}\right)_{s}$

$\rho_{n f}=(1-\varphi) \rho_{f}+\varphi \rho_{s}, \mu_{n f}=\frac{\mu_{f}}{(1-\varphi)^{2.5}}$,

$\frac{k_{n f}}{k_{f}}=\frac{\left(k_{s}+2 k_{f}\right)-2 \varphi\left(k_{f}-k_{s}\right)}{\left(k_{s}+2 k_{f}\right)+\varphi\left(k_{f}-k_{s}\right)}$,

Where $\mu_{f}$ is the viscosity of the fluid fraction, $\varphi$ is volume fraction of nanofluids, $\rho_{f}$ and $\rho_{s}$ are the density of the liquid fraction and a solid fraction, respectively. It should be noted that the above equation is to calculate $k_{n f}$ limited spherical nanoparticles and is not used for other geometries nanofluids relationship and the relationship will change. Thermo-physical properties of the base fluid (water) and various particles are given in Table 1 [16].

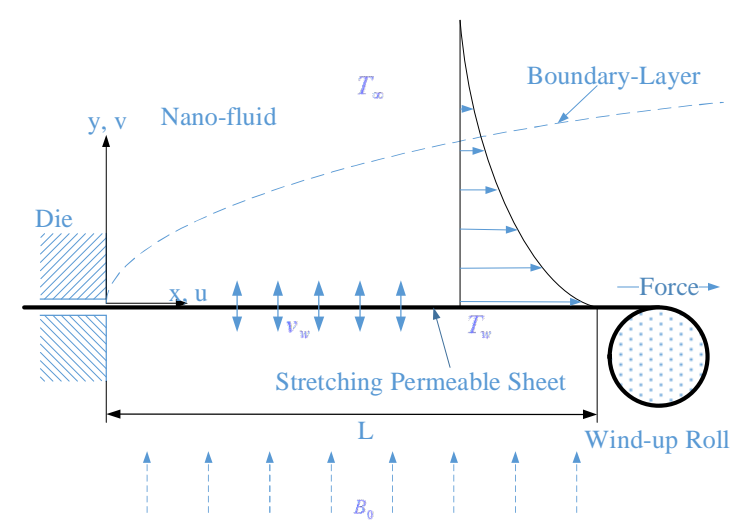

Fig. 1: The current configuration on page spread and coordinate the issue

Table 1: thermo-physical properties different base fluid and particles [16]

\begin{tabular}{cccc}
\hline Thermal conductivity & Density & Specific heat at constant pressure & Physical properties \\
\hline 0.613 & 997.1 & 4179 & Phase of fluid (water) \\
400 & 8933 & 385 & Copper \\
76.5 & 6320 & 531.8 & Copper oxide \\
40 & 3970 & 765 & Aluminium oxide \\
\hline
\end{tabular}


Boundary conditions to issue the uniform suction of the page is as follows:

$\left.\begin{array}{l}u=u_{w}, \\ v=v_{0}, \\ T=T_{w}(x)=T_{\infty}+b x,\end{array}\right\}$ at $y=0$,
$\left.\begin{array}{l}u \rightarrow 0, \\ T \rightarrow T_{\infty},\end{array}\right\}$ as $y \rightarrow \infty$,

In the above equation is a constant parameter. By introducing the following $\psi(x, y)$ dimensionless flow, continuity equation will be satisfied:

$u=\frac{\partial \psi}{\partial y}=a x f^{\prime}(\eta)$

$v=-\frac{\partial \psi}{\partial x}=-\left(v_{f} a\right)^{1 / 2} f(\eta)$,

The dimensionless components of velocity and temperature distribution conservation equations (1) to (3), using a similar and parallel definition of $\eta$ parameter definitions are as follows:

$$
\begin{aligned}
& \eta=\left(a / v_{f}\right)^{1 / 2} y, \\
& \psi=\left(v_{f} a\right)^{1 / 2} x f(\eta), \\
& \theta(\eta)=\frac{T-T_{\infty}}{T_{w}-T_{\infty}},
\end{aligned}
$$

Using equation (4) and as well as a parallel transformation equation and replacing them in equation (1) to (3), the following system of ordinary differential equations is:

$$
\begin{aligned}
& \frac{1}{(1-\varphi)^{2.5}} f^{\prime \prime \prime}-M f^{\prime}+ \\
& \left(1-\varphi+\varphi \frac{\rho_{s}}{\rho_{f}}\right)\left(f f^{\prime \prime}-f^{\prime 2}\right)=0, \\
& \frac{k_{n f} / k_{f}}{1-\varphi+\varphi\left(\left(\rho c_{p}\right)_{s} /\left(\rho c_{p}\right)_{f}\right)} \theta^{\prime \prime}+ \\
& \operatorname{Pr}\left(f \theta^{\prime}-f^{\prime} \theta\right)=0,
\end{aligned}
$$

In the above equations, $M=\sigma B_{0}^{2} / a \rho_{f}$ is the magnetic parameter and $\operatorname{Pr}=v_{f}\left(\rho c_{p}\right)_{f} / k_{f}$ is Prandtl number. The transformed boundary conditions are as follows: 


$$
\left.\begin{array}{l}
\left.\begin{array}{l}
f(\eta)=f_{w}, \\
f^{\prime}(\eta)=1, \\
\theta(\eta)=1,
\end{array}\right\} \text { at } \quad \eta=0, \\
f^{\prime}(\eta) \rightarrow 0, \\
\theta(\eta) \rightarrow 0,
\end{array}\right\} \text { as } \quad \eta \rightarrow \infty,
$$

Where $f_{w}=-v_{0} / \sqrt{v_{f} a}$ is the suction parameter.

\section{Review entropy production}

According to references [1] and [17], the rate of local entropy production volume in the presence of a uniform magnetic field with a view to simplifying assumptions boundary layer is derived as follows:

$$
\begin{aligned}
\dot{S}_{\text {gen }}^{\prime \prime \prime} & =\frac{k_{n f}}{T_{\infty}^{2}}\left[\left(\frac{\partial T}{\partial x}\right)^{2}+\left(\frac{\partial T}{\partial y}\right)^{2}\right] \\
+ & \frac{\mu_{n f}}{T_{\infty}}\left(\frac{\partial u}{\partial y}\right)^{2}+\frac{\sigma B_{0}^{2}}{T_{\infty}} u^{2},
\end{aligned}
$$

The above equation shows that the entropy production is consisted of three parts: the first part, the effects of conductivity, which is proportional to the local entropy production due to the irreversibility of heat transfer. This includes the production of entropy due to the heat conduction of the sheet. The flow of electrical conductivity is induced in the presence of an external magnetic field of fluid flow force, this force can cause moving fluid. Entropy production number that entropy production rate is dimensionless form, represents the ratio between $\left(\dot{S}_{g e n}^{\prime \prime \prime}\right)$ the actual rate of entropy and entropy production rate. Parallel conversion parameters (6) and (7) to non-dimensional equation of entropy (11) is used as a result of entropy production number of the equation comes in the form below:

$$
\begin{aligned}
& N_{G}=\frac{k_{n f}}{k_{f}}\left(\frac{1}{X^{2}} \theta(\eta)^{2}+\operatorname{Re}_{L} \theta^{\prime}(\eta)^{2}\right)+ \\
& \frac{1}{(1-\varphi)^{2.5}} \frac{B r \operatorname{Re}_{L}}{\Omega} f^{\prime \prime}(\eta)^{2}+\frac{B r H a^{2}}{\Omega} f^{\prime}(\eta)^{2},
\end{aligned}
$$

Where $B r=\frac{\mu_{f} u_{p}^{2}}{k_{f} \Delta T}=\frac{\mu_{f} a^{2} x}{k_{f} b}$ is $\quad$ Brinkman number, $\operatorname{Re}_{L}=\frac{u_{L} L}{v_{f}}=\frac{a L^{2}}{v_{f}}$ is Reynolds number, $X=\frac{x}{L}$ is dimensionless longitudinal coordinate, $H a=B_{0} L \sqrt{\frac{\sigma}{\mu_{f}}}$ is the Hartmann number, 
$\Omega=\Delta T / T_{\infty}$ is a dimensionless parameter temperature difference and $\dot{S}_{0}^{\prime \prime \prime}=\frac{k_{f} \Delta T^{2}}{L^{2} T_{\infty}^{2}}=\frac{k_{f} b^{2} x^{2}}{L^{2} T_{\infty}^{2}}$ is the rate of entropy production are characteristic.

\section{Discussion and Results}

Nonlinear ordinary differential equations (8) and (9) are solved according to the boundary conditions (10) for Runge-Kutta numerical method for different values of magnetic parameters, the volume fraction of nanoparticles, and suction. In this section, three types of nanoparticles have been considered: copper, copper oxide, aluminum oxide. Water is considered as a base fluid. It should be noted that copper, $C u$, in all forms, but the forms that exist to review the types of nanoparticles, as nanoparticles are considered. Prandtl number equal to 2.6 that represents the Prandtl number of water is considered at $20^{\circ} \mathrm{C}$ [16].

The comparison between some of the results in this article and previously published papers [18-21] is shown in Table 2 that the results show excellent agreement between the results obtained is.

Table 2: Comparison of the results for different values of Prandtl number on the transmission

$$
\operatorname{rate} \varphi=M=f_{w}=0
$$

\begin{tabular}{cccccc}
\hline current study & Ref. [21] & Ref. [20] & Ref. [19] & Ref. [18] & Prandtl number \\
\hline 0.8086 & 0.8086 & 08086 & 0.8086 & 0.8058 & 0.72 \\
1.9236 & 1.9236 & 1.9237 & 1.9237 & 1.9144 & 3 \\
3.07225 & 3.07224 & 3.0723 & 3.0723 & 3.7006 & 7 \\
3.7206 & 3.7206 & 3.7207 & 3.7207 & - & 10 \\
\hline
\end{tabular}

Fig.s 2 and 3, involved to examine the effect of the volume fraction of nanoparticles on the curve of speed and temperature distribution curve. The results show that with increasing volume fraction of nanoparticles, the velocity component decreases. This phenomenon occurs because the nanoparticles leads to further thinning-boundary layer thickness os speed. On the other hand, with increasing volume fraction of nanoparticles, thermal conductivity, thermal boundary layer thickness and consequently increases.

Fig.s 4 and 5 show the magnetic parameters effects on velocity and also temperature distribution curve. Such as drag force, called the Lorentz force arises due to the magnetic field applied perpendicular to the flow of electrical conductivity. This force tends to decrease with increasing temperature and flow rate near the screen. Therefore, the amount of speed reduction and thermal boundary layer thickness-increasing magnetic parameter increases. It should be noted that a large resistance on the fluid particles generate heat in the fluid, caused by increased magnetic parameters.

Fig.s 6 and 7 show a suction parameter effect on the speed distribution curve and air distribution curve. When the vacuum is created in the suction wall, speed curve is reduced, 
because the amount of fluid in the suction pulled into the walls, boundary layers will be thinner. Gradual reduction in the thermal boundary layer thickness is created for large-suction parameter.

Fig.s 8 to 11 show the results of entropy production number for the parameters of volume fraction of nanoparticles, magnetic, suction, and various nanoparticles. It is observed that parameter occurs with increasing volume fraction of nanoparticles, magnetic parameters, and parameter suction entropy production rate increases. Also, the observation is made that in most parts of the boundary layer formed around the sheet, nano-fluids with copper nanoparticle production is the highest entropy.

Fig.s 12 to 14 represent the effect of Reynolds numbers, Brinkman and Hartmann entropy production number. The results show an increase in entropy production number with increasing Reynolds number, Brinkman number and Hartmann number. As the equation (12) determined, with increasing Reynolds number, irreversibility due to heat transfer and fluid friction surface and consequently lead to an average increase of entropy. Also, there is an increasing number Brinkman, entropy production due to Joule dissipation and increases fluid friction and ultimately increasing Hartmann number entropy production increases due to Joule dissipation and consequently the average entropy production. As can be seen from the increase in value of the Reynolds number $\operatorname{Re}=10$ and $\operatorname{Re}=50$ (5-fold change), and entropy production number on the screen from $\mathrm{NG}=200$ to $\mathrm{NG}=1000$ (5-fold increase) changes. On the other hand, with 5 times the amount of entropy production number changes on the screen Brinkman number of $\mathrm{NG}=200$ up to $\mathrm{NG}=900$. Finally, it is clear that with increasing Hartmann number of $\mathrm{Ha}=1$ to $\mathrm{Ha}=15$ (15-fold change), the amount of entropy production number changes on the screen from $\mathrm{NG}=200$ up to $\mathrm{NG}=1000$. Therefore, by comparing the results it is clear that in the area studied in this paper, the highest rate of entropy production number is obtained for the change in the Reynolds number.

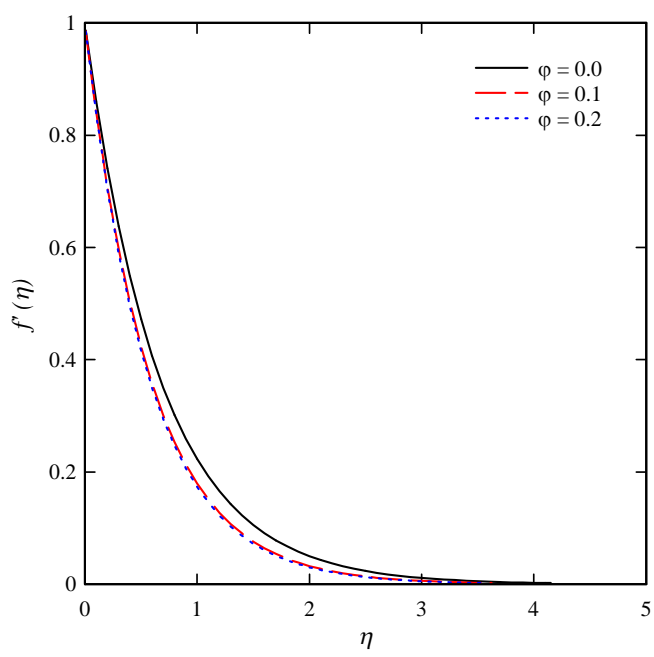

Fig. 2: Effect of particle volume fraction on the velocity curve for $M=f_{w}=0.5$ 
Bulletin de la Société Royale des Sciences de Liège, Vol. 85, 2016, p. 47 - yy

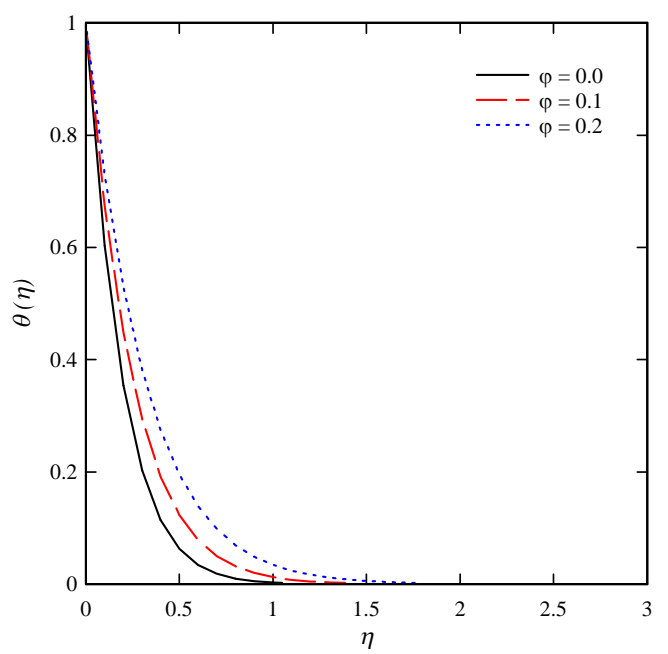

Fig. 3: Effect of particle volume fraction on the temperature distribution curve for $M=f_{w}=0.5$

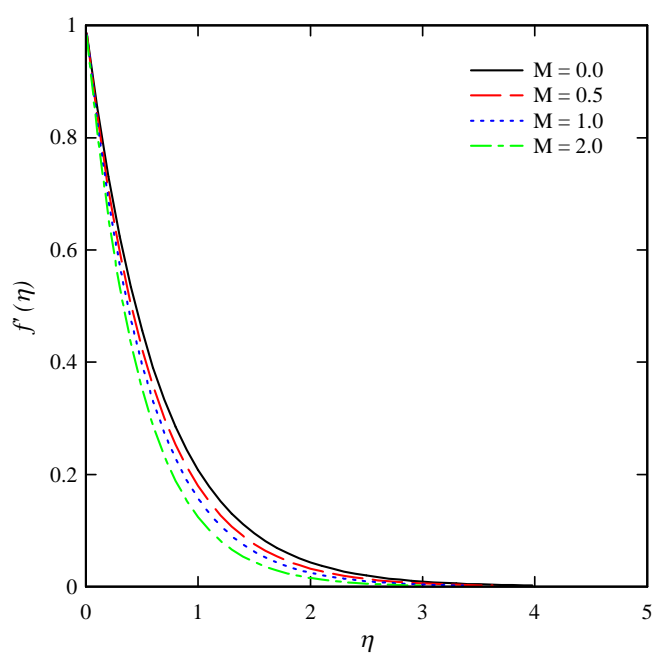

Fig. 4: Effect of magnetic parameters on a velocity curve for $\varphi=0.1, f_{w}=0.5$

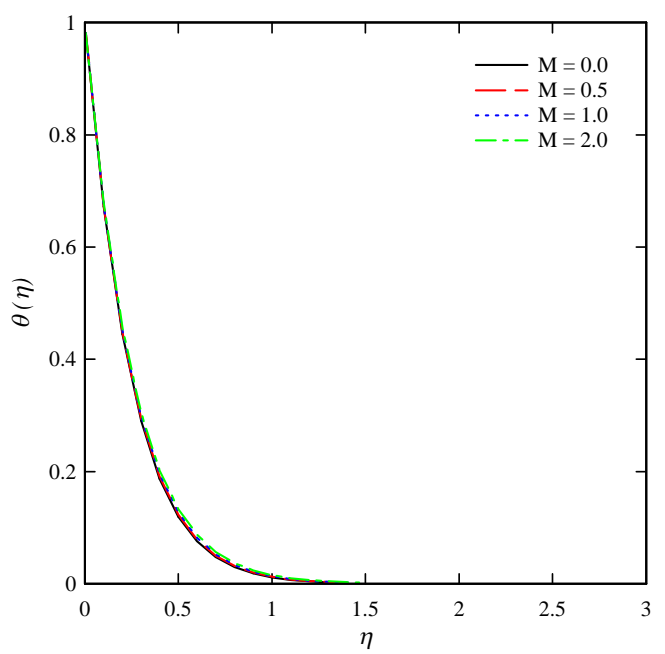

Fig. 5: Effect of magnetic parameters on temperature distribution curve for $\varphi=0.1, f_{w}=0.5$ 
Bulletin de la Société Royale des Sciences de Liège, Vol. 85, 2016, p. 47 - yy

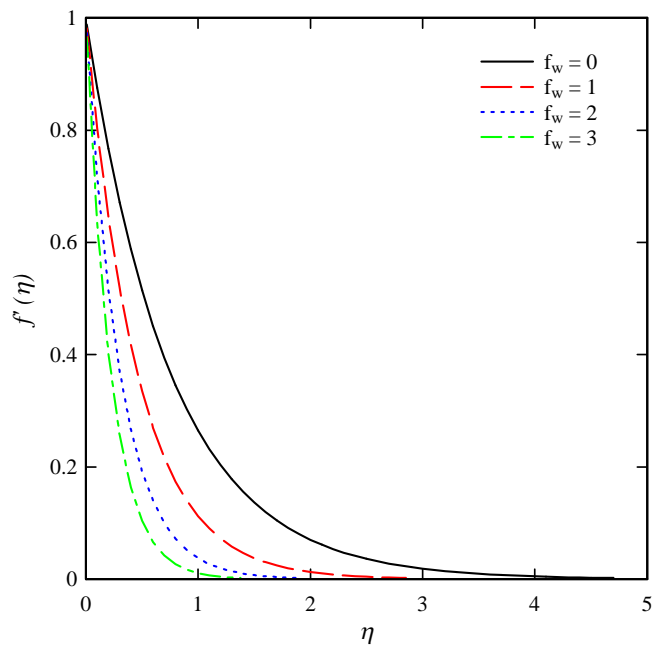

Fig. 6: Effect of suction on the velocity curve for $\varphi=0.1, M=0.5$

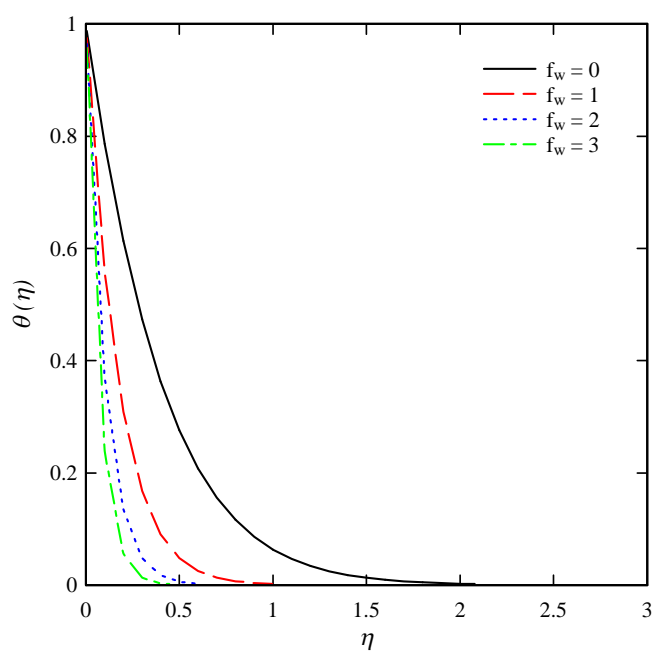

Fig. 7: Effect of suction on the temperature distribution curve for $\varphi=0.1, M=0.5$

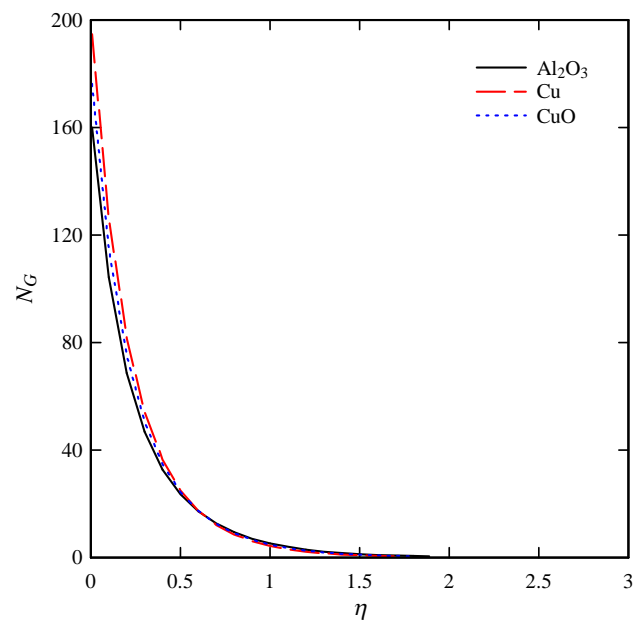

Fig. 8: The effect of different nanoparticles on entropy production number for $\varphi=0.1, M=f_{w}=0.5, H a=1, B r=R e=10$ 
Bulletin de la Société Royale des Sciences de Liège, Vol. 85, 2016, p. 47 - yy

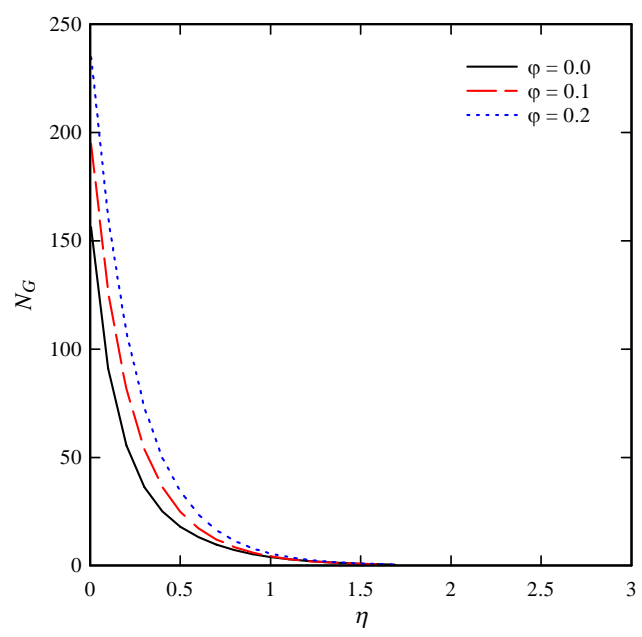

Fig. 9: Effect of volume fraction of nanoparticles on the number of entropy for $M=f_{w}=0.5, H a=1, B r=R e=10$

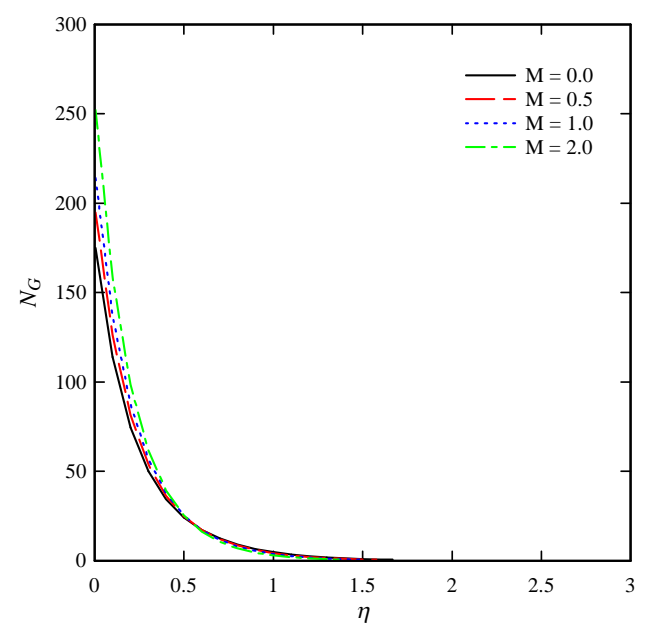

Fig. 10: Influence of magnetic parameters on the number of entropy for $\varphi=0.1, f_{w}=0.5, H a=1, B r=R e=10$

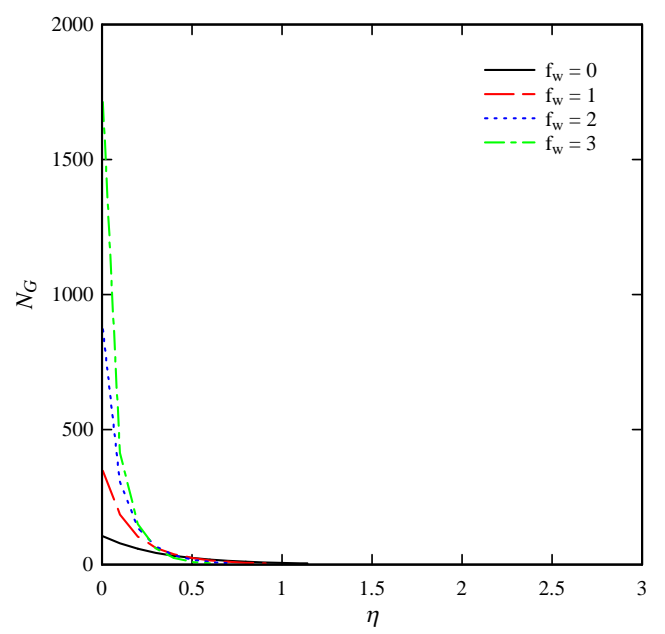

Fig. 11: Effect of suction on the number of entropy for $\varphi=0.1, M=0.5, H a=1, B r=R e=10$ 
Bulletin de la Société Royale des Sciences de Liège, Vol. 85, 2016, p. 47 - yy

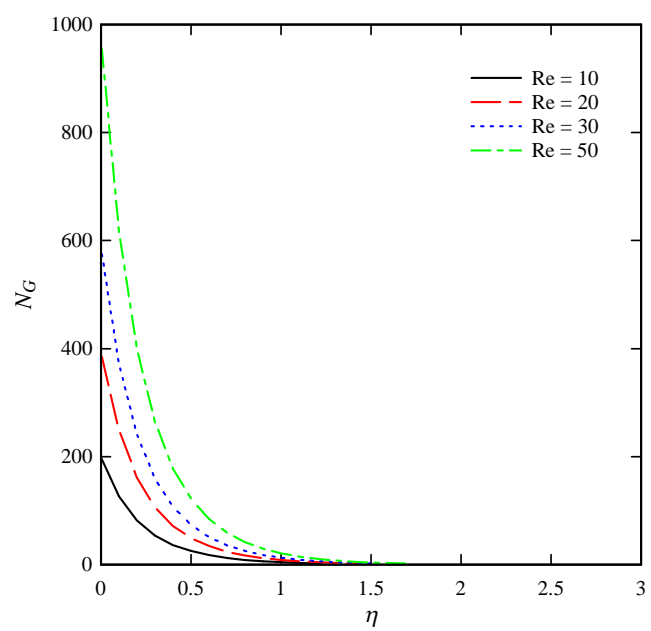

Fig. 12: Influence of Reynolds number on the number for the entropy production for

$$
\varphi=0.1, M=f_{w}=0.5, H a=1, B r=10
$$

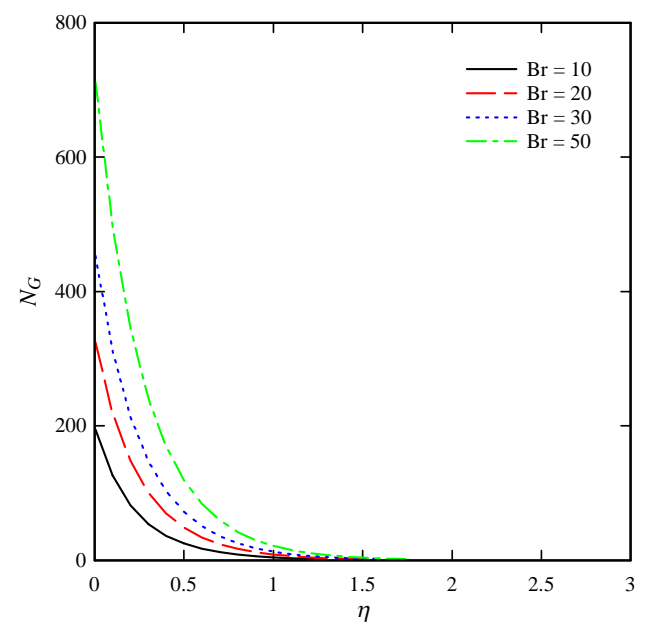

Fig. 13: Impact of Brinkman number on entropy production number for

$$
\varphi=0.1, M=f_{w}=0.5, H a=1, R e=10
$$

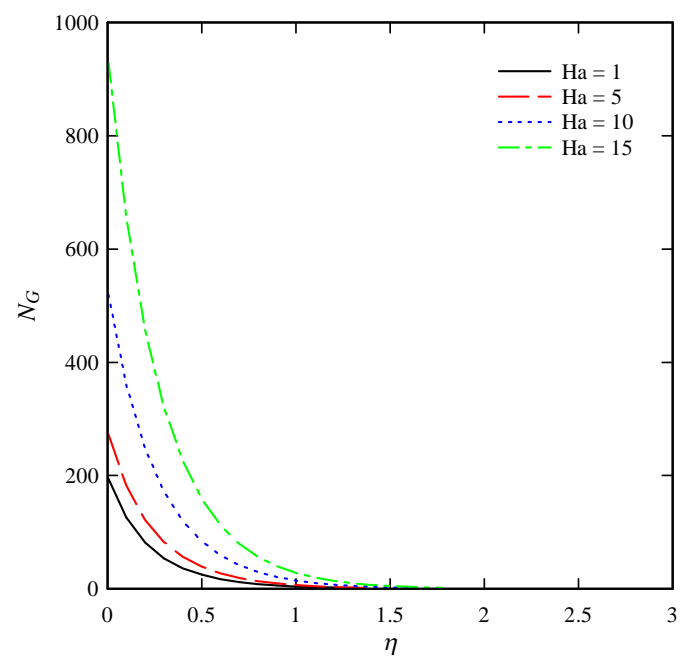

Fig. 14: Influence of Hartmann number on the number for the entropy production

$$
\varphi=0.1, M=f_{w}=0.5, B r=R e=10
$$




\section{Conclusion}

In this study, fluid flow, heat transfer and entropy production in nanofluids on page spread permeable slip boundary condition will be discussed in the presence of magnetic hydrodynamic flow. Conservation equations in the form of partial differential equations, ordinary differential equations, which is used in order to convert them to a parallel solution for speed components as well as non-dimensional temperature distribution. Dimensionless ordinary differential equations obtained numerically have been resolved using Runge-Kutta and delivery techniques. The results obtained in this study, other references and also used analytical solutions, which well be seen that shows the accuracy of the solution is in this article. With increasing volume fraction of nanoparticles, the amount of components to reduce speed and hydrodynamic boundary layer thickness and thermal conductivity and thus the thermal boundary layer thickness-increased. Also, the components and reduce the speed of the boundary layer and thermal boundary layer thickness-increasing magnetic parameter increases.

\section{References}

[1] A. Bejan, Second law analysis in heat transfer, Energy 5 (1980) 720-732 .

[2] A. A. Bejan, Entropy generation minimization: The method of thermodynamic optimization of finite-size systems and finite-time processes, CRC PressINC, 1996.

[3] A. Bejan, A Study of Entropy Generation in Fundamental Convective Heat Transfer, Journal of Heat Transfer 101 (1979) 718-725.

[4] S. Mahmud, R. A. Fraser, The second law analysis in fundamental convective heat transfer problems, International Journal of Thermal Sciences 42 (2003) 177-186.

[5] B. S. Yilbas, Entropy analysis of concentric annuli with rotating outer cylinder, Exergy, An International Journal 1 (2001) 60-66.

[6] S. Mahmud, R. A. Fraser, Second law analysis of heat transfer and fluid flow inside a cylindrical annular space, Exergy, An International Journal 2 (2002) 322-329.

[7] S. Mahmud, R. A. Fraser, Analysis of entropy generation inside concentric cylindrical annuli with relative rotation, International Journal of Thermal Sciences 42 (2003) 513-521.

[8] M. Mirzazadeh, A. Shafaei, F. Rashidi, Entropy analysis for non-linear viscoelastic fluid in concentric rotating cylinders, International Journal of Thermal Sciences 47 (2008) 1701-1711.

[9] S. U. S. Choi, J. A. Eastman, Enhancing thermal conductivity of fluids with nanoparticles, Materials Science 231 (1995) 99-105.

[10] J. A. Eastman, U. S. Choi, S. Li, G. Soyez, L. J. Thompson, R. J. DiMelfi, Novel Thermal Properties of Nanostructured Materials, Materials Science Forum 312 - 314 (1999) 629-634.

[11] Y . Xuan, W. Roetzel, Conceptions for heat transfer correlation of nanofluids, International Journal of Heat and Mass Transfer 43 (2000) 3701-3707.

[12] M. Mustafa, T. Hayat, I. Pop, S. Asghar, S. Obaidat, Stagnation-point flow of a nanofluid towards a stretching sheet, International Journal of Heat and Mass Transfer 54 (2011) 5588-5594.

[13] S. Khalili, S. Dinarvand, R. Hosseini, H. Tamim, I. Pop, Unsteady MHD flow and heat transfer near stagnation point over a stretching/shrinking sheet in porous medium filled with a nanofluid, Chinese Physics B 23 (2014) 048203.

[14] M. Ziaei-Rad, A. Kasaeipoor, A Numerical study of similarity solution for mixed-convection copper-water nanofluid boundary layer flow over a horizontal plate, Modares Mechanical Engineering 14 (2015) 190-198 .

[15] H. C. Brinkman, The Viscosity of Concentrated Suspensions and Solutions, The Journal of Chemical Physics 20 (1952) 571-571.

[16] H. Oztop, E. Abu-Nada, Numerical study of natural convection in partially heated rectangular enclosures filled with nanofluids, International Journal of Heat and Fluid Flow 29 (2008) 1326-1336. 
Bulletin de la Société Royale des Sciences de Liège, Vol. 85, 2016, p. 47 - yy

[17] S. Aïboud, S. Saouli, Entropy analysis for viscoelastic magnetohydrodynamic flow over a stretching surface, International Journal of Non-Linear Mechanics 45 (2010) 482-489

[18] M. E. Ali, Heat transfer characteristics of a continuous stretching surface, Wärme - und Stoffübertragung 29 (1994) 227-234 .

[19] A. Ishak, R. Nazar, I. Pop, Boundary layer flow and heat transfer over an unsteady stretching vertical surface, Meccanica 44 (2009) 369-375.

[20] L. J. Grubka, K. M. Bobba, Heat Transfer Characteristics of a Continuous, Stretching Surface With Variable Temperature, Journal of Heat Transfer 107 (1985) 248-250.

[21] A. Mahdy, Unsteady mixed convection boundary layer flow and heat transfer of nanofluids due to stretching sheet, Nuclear Engineering and Design 249 (2012) 248-255 . 